PROCEEDINGS OF THE

AMERICAN MATHEMATICAL SOCIETY

Volume 43, Number 2, April 1974

\title{
EILENBERG-MAC LANE SPECTRA
}

\author{
H. R. MARGOLIS ${ }^{1}$
}

Abstract. Let $K\left(Z_{p}\right)$ be the Eilenberg-Mac Lane spectrum with homotopy $Z_{p}$ and let $A=H^{*}\left(K\left(Z_{p}\right) ; Z_{p}\right)$-the $\bmod p$ Steenrod algebra. Let $X$ be a locally finite spectrum. It is proven that

$$
\left[K\left(Z_{p}\right), X\right] \rightarrow \operatorname{Hom}_{A}\left(H^{*}\left(X ; Z_{p}\right), A\right)
$$

is an isomorphism. It is also proven that there is a unique decomposition $X=\left(\oplus K\left(Z_{p}\right)\right) \oplus Y$ where $H^{*}\left(Y ; Z_{p}\right)$ as an $A$-module has no free summands.

0. Introduction. The primary purpose of this paper is to demonstrate the special role of Eilenberg-Mac Lane spectra in stable homotopy theory. Let $K\left(Z_{p}\right)$ denote the Eilenberg-Mac Lane spectrum associated to $Z_{p}$. It is the spectrum defining $\bmod p$ cohomology denoted $H^{*}\left(; Z_{p}\right)$ and its algebra of self-maps-the algebra of $\bmod p$ stable cohomology operations-is the mod $p$ Steenrod algebra $A$. It is trivially true that the homomorphism $\left[X, K\left(Z_{p}\right)\right] \rightarrow \operatorname{Hom}_{A}\left(H^{*}\left(K\left(Z_{p}\right) ; Z_{p}\right), H^{*}\left(X ; Z_{p}\right)\right)$, which assigns to each map its induced map in cohomology, is an isomorphism. But the algebra $A$ possesses some rather special properties (see [1], [4], [5]) and because of this we can prove in addition:

THEOREM. If $X$ is bounded below and locally finite then the homomorphism

$$
\left[K\left(Z_{p}\right), X\right] \rightarrow \operatorname{Hom}_{A}\left(H^{*}\left(X ; Z_{p}\right), H^{*}\left(K\left(Z_{p}\right) ; Z_{p}\right)\right)
$$

is an isomorphism.

We will also prove two parallel results, one algebraic and one topological, that further elucidate the special role of $K\left(Z_{p}\right)$.

THEOREM. Let $M$ be a bounded below A-module. Then $M$ is isomorphic to $N \oplus F$ where $F$ is a free A-module and $N$ has no free summands, and, further, this decomposition is unique up to isomorphism.

Let $X \oplus Y$ denote direct sum in the stable homotopy category.

Received by the editors May 30, 1973 and, in revised form, July 23, 1973.

AMS (MOS) subject classifications (1970). Primary 55D20, 55B20; Secondary $55 \mathrm{G} 10$.

Key words and phrases. Eilenberg-Mac Lane spectra, Steenrod algebra.

${ }^{1}$ The author was partially supported by NSF grant 28565 .

(c) American Mathematical Society 1974 
THEOREM. Let $X$ be a bounded below, locally finite spectrum. Then $X$ is equivalent to $Y(\oplus K V)$ where $V$ is a graded $Z_{p}$-vector space and $H^{*}\left(Y ; Z_{p}\right)$ has no free summands, and further this decomposition is unique up to equivalence.

1. Modules over algebras like the Steenrod algebra. Recall that a Poincare algebra $B$ over $R$ is a connected algebra such that, for some $n$, there is an $R$-map $f: B_{n} \rightarrow R$ for which the bilinear pairing $B_{r} \times B_{n-r} \rightarrow R$ defined by $(a, b) \rightarrow f(a b)$ is nonsingular [5].

Let $A$ be a connected algebra over a field which satisfies the following conditions:

(a) $A$ is the union of a directed system of subalgebras $\left\{A_{n}\right\}, n \in I$, such that each $A_{n}$ is a Poincaré algebra.

(b) $A$ is flat as an $A_{n}$-module for each $n$.

EXAMPLES. (1) If $A$ is a connected Hopf algebra which is the union of finite sub-Hopf algebras, then $A$ is such an algebra (see [4] or [5]).

(2) In particular if $A$ is the $\bmod p$ Steenrod algebra then it is such an algebra.

(3) If $A$ is a connected exterior algebra on generators $\left\{x_{\alpha}\right\}, \alpha \in \Lambda$, then $A$ is such an algebra where $I$ is the directed set of finite subsets of $\Lambda$ and, for $n \in I, A_{n}$ is the exterior algebra generated by $x_{\alpha}$ with $\alpha \in n$.

Let $A$ be as above and let $\mathscr{M}_{A}$ be the category of bounded below $A$-modules (a $Z$-graded object $M_{*}$ is bounded below if there is an $m$ such that, for all $i \leqq m, M_{i}=0$ ).

Theorem. If $F$ is a free A-module in $\mathscr{M}_{A}$ then $F$ is injective in $\mathscr{M}_{A}$.

This result was originally proven in the case in which $A$ is the mod 2 Steenrod algebra by J. F. Adams and the author [1]. The general result is due to Moore and Peterson [5] - their version is somewhat more general than that stated here-and a different proof can be found in [4]. As an immediate corollary we have:

Corollary. In $\mathscr{M}_{A}$ if $F$ is free then $\operatorname{Hom}_{A}(, F)$ is exact.

A further instance of the particularly simple role of free modules in $\mathscr{M}_{A}$ is the following:

THeorem 1. For any module $M$ in $\mathscr{M}_{A}, M$ is isomorphic to $N \oplus F$ where $F$ is a free $A$-module and $N$ has no free summands, and further this decomposition is unique up to isomorphism.

Proof. With no assumptions on the algebra $A$ it is easily shown by the usual transfinite methods that there is an exact sequence $0 \rightarrow F \rightarrow M \rightarrow$ $N \rightarrow 0$ where $F$ is free and $N$ has no free summands. But $F$ is injective in $\mathscr{M}_{A}$ and therefore the sequence splits. 
This gives the desired decomposition and we will now show that it is unique up to isomorphism. Suppose that we have an isomorphism $\alpha: N_{1} \oplus F_{1} \rightarrow N_{2} \oplus F_{2}$ with $F_{1}$ and $F_{2}$ free, and $N_{1}$ and $N_{2}$ having no free summands. Let $j: F_{1} \rightarrow N_{2} \oplus F_{2}$ be the composite $\alpha i$ where $i$ is the canonical inclusion. Then we have $0 \rightarrow F_{1} \rightarrow N_{2} \oplus F_{2} \rightarrow{ }^{k} N_{3} \rightarrow 0$ exact and $\alpha$ induces an isomorphism of $N_{1}$ and $N_{3}$. Let $f: F_{1} \rightarrow F_{2}$ be the composite $\Pi j$ where $\Pi$ is the projection and let $g: N_{2} \rightarrow N_{3}$ be the composite $k l$ where $l$ is the canonical inclusion. We will prove that $f$ is an isomorphism and the following commutative diagram then implies that $g$ is an isomorphism:

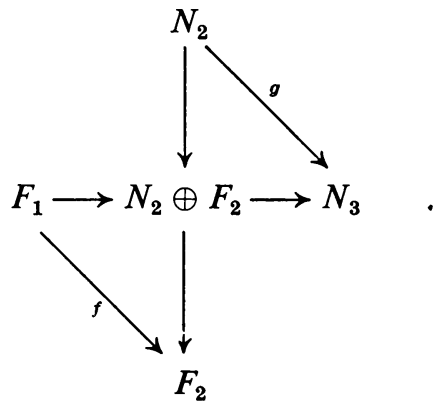

We first prove that $f$ is monic. Let $F_{1}$ have an $A$-base $\left\{x_{i}\right\}$ and $F_{2}$ an $A$-base $\left\{y_{j}\right\}$ and suppose that $f\left\{\sum a_{i} x_{i}\right)=0$. If $j\left(x_{i}\right)=\left(m_{i}, z_{i}\right)$ then this implies that $j\left(\sum a_{i} x_{i}\right)=\left(\sum a_{i} m_{i}, 0\right)$. Our assumption that $N_{2}$ has no free summands implies that, for each $m_{i}$, there is a $b_{i} \neq 0$ in $A$ such that $b_{i} m_{i}=0$. Since the summation is finite $a_{i}, b_{i} \in A_{n}$ for some $n$ and all $i$. Since $A_{n}$ is a Poincaré algebra there is an element $c$ in $A_{n}$ such that $c a_{i} m_{i}=0$ for all $i$ and $c a_{i} \neq 0$ for some $i$. Then $c \sum a_{i} x_{i} \neq 0$ but $j\left(c \sum a_{i} x_{i}\right)=0$, which is a contradiction.

To show that $f$ is epic it will suffice to show that $y_{j}$ is in the image of $f$ for all $j$. Suppose that $y_{k} \notin \operatorname{im} f$ for some $y_{k}$ which we may assume to have minimal degree with this property. In any case $\left(0, y_{k}\right)=\alpha(n, x)$ which implies that $\alpha(n, 0)=\left(m, y^{\prime}\right)$ and $\alpha(0, x)=\left(m, y^{\prime \prime}\right)$ with either

or

$$
y^{\prime}=y_{k}+\sum_{j \neq k} a_{j} y_{j} \text { and } y^{\prime \prime}=\sum_{j \neq k} a_{j} y_{j} \text {, }
$$

$$
y^{\prime}=\sum_{j \neq k} a_{j} y_{j} \quad \text { and } y^{\prime \prime}=y_{k}+\sum_{j \neq k} a_{j} y_{j} .
$$

In the first case since $N_{1}$ has no free summands there is an $a \neq 0$ in $A$ such that $a n=0$, in which case we have $0=\alpha(a n, 0)=\left(a m, a y^{\prime}\right) \neq 0$. In the second case either $\operatorname{deg} a_{j}>0$ for all $j \neq k$ and, therefore, by our minimality assumption, $y_{j} \in \operatorname{im} f$ for $j \neq k$-but this implies that $y_{k} \in \operatorname{im} f$, or $\operatorname{deg} a_{j}=0$ for some $j \neq k$, and we can argue as we did in the first case. 
2. Eilenberg-Mac Lane spectra. Let $\mathscr{T}$ be the homotopy category of bounded below spectra constructed by Boardman [2], [3], [6] (in his terminology "highly connected" spectra). We will not need Boardman's construction of the objects and maps of $\mathscr{T}$ but we will use a number of the formal properties of this category, explicitly:

(a) $\mathscr{T}$ is additive and triangulated.

(b) If $f: X \rightarrow Y$ induces an isomorphism of homotopy or integral cohomology then $f$ is an equivalence.

(c) If there is a common bound to the connectivity of $X_{\alpha}, \alpha \in \Lambda$, then the direct sum $\oplus X_{\alpha}$ exists in $\mathscr{T}$.

(d) There is a functor $K: \mathscr{A} b \rightarrow \mathscr{T}$ which assigns to each abelian group $G$ an Eilenberg-Mac Lane spectrum $K(G)$, this functor preserves sums, takes short exact sequences to exact triangles and induces an isomorphism $\operatorname{Hom}(G, H) \rightarrow[K(G), K(H)]^{\circ}$.

(e) For $X$ in $\mathscr{T}$ there is a Postnikov tower $X_{0} \leftarrow X_{1} \leftarrow \cdots \leftarrow X$ and for $Y$ in $\mathscr{T},[Y, X] \rightarrow \operatorname{inj} \lim \left[Y, X_{r}\right]$ is onto.

A spectrum $X$ is locally finite if $\Pi_{r}(X)$ is finitely generated for each $r$.

Fix a prime $p$ and let $H^{*}(X)=\left[X, K\left(Z_{p}\right)\right]^{*}, \bmod p$ cohomology, which we regard as a left module over $A=H^{*}\left(K\left(Z_{p}\right)\right)$, the $\bmod p$ Steenrod algebra. For a graded (bounded below) $Z_{p}$-vector space $V$, let $K(V)=$ $\bigoplus_{\alpha} \Sigma^{d_{\alpha}} K\left(Z_{p}\right)$ where $V$ has a basis $\left\{x_{\alpha}\right\}$ with $\operatorname{deg} x_{\alpha}=d_{\alpha}$.

The topological results of this paper are:

THEOREM 2. (a) If $X$ is locally finite and $\alpha: H^{*}(X) \rightarrow N \oplus F$ is an isomorphism of A-modules with $F$. free over $A$ then there are spectra $Y$ and $K(V)$ such that $H^{*}(Y)=N, H^{*}(K(V))=F$ and there is an equivalence $k: Y \oplus K(V) \rightarrow X$ such that $k^{*}=\alpha$.

(b) If $X$ is locally finite then it is equivalent to $Y \oplus K(V)$ where $H^{*}(Y)$ has no free summands, and then $Y$ and $K(V)$ are unique (up to equivalence).

THEOREM -3. If $X$ is locally finite then

$$
[K(V), X] \rightarrow \operatorname{Hom}_{A}\left(H^{*}(X), H^{*}(K(V))\right)
$$

is an isomorphism.

Note. (1) The restriction on $X$ in Theorem 2(a) is essential. Let $X=K\left(Z_{p}\right) \oplus K\left(Z_{p}\right) \oplus \cdots$ then $H^{*}(X)=A \times A \times \cdots$ and $F=A \oplus A \oplus \cdots$ is a direct summand of $H^{*}(X)$ since free $A$-modules are injective in $\mathscr{M}_{A}$. But we observe that $F$ is not realizable for, if $F=H^{*}(W)$, then

$$
\operatorname{Hom}\left(H_{\circ}(W), Z_{p}\right)=H^{\circ}(W)=Z_{p} \oplus Z_{p} \oplus \cdots,
$$

which cannot occur.

(2) I do not know if the restriction on $X$ in Theorem 2(b) is essential.

(3) The restriction on $X$ in Theorem 3 is essential. Let $X$ be as in 
Note (1), then the map $\left[K\left(Z_{p}\right), X\right] \rightarrow \operatorname{Hom}_{A}\left(H^{*}(X), A\right)$ is not an isomorphism. For we have that $\left[K\left(Z_{p}\right), X\right]^{\circ}=\operatorname{Hom}\left(Z_{p}, Z_{p} \oplus Z_{p} \oplus \cdots\right)$ which is countable but $\operatorname{Hom}_{A}^{\circ}\left(H^{*}(X), A\right)=\operatorname{Hom}_{A}^{\circ}(A \times A \times \cdots, A)$ which is uncountable. Note however that $\left[X, K\left(Z_{p}\right)\right] \rightarrow \operatorname{Hom}_{A}\left(A, H^{*}(X)\right)$ is an isomorphism.

Theorems 2 and 3 will be proven by the following sequence of steps.

STEP. 1. We prove Theorem 3 for spectra with finite Postnikov towers and from this prove that the map in Theorem 3 is epic in general.

STEP 2. We prove Theorem 2 using the results of Step 1.

STEP 3. We complete the proof of Theorem 3 using the results of Step 2.

STEP 1. Let $F=H^{*}(K(V))$, it is both projective and injective in $\mathscr{M}_{A}$. We begin by proving Theorem 3 for $X=K(G)$ where $G$ is $Z_{p}, Z_{q}$ for $q \neq p$ a prime or $Z$. The first case is immediate from the definitions of $H^{*}$ and $A$. The second case follows from the fact that for $p$ and $q$ distinct primes $H^{*}\left(K\left(Z_{q}\right) ; Z_{p}\right)=0$. The third case is slightly more involved. From the short exact sequence $0 \rightarrow Z \rightarrow Z \rightarrow Z_{p} \rightarrow 0$ we get the exact triangle $K(Z) \rightarrow K(Z) \rightarrow K\left(Z_{p}\right) \rightarrow K(Z)$ and from this we get the following diagram:

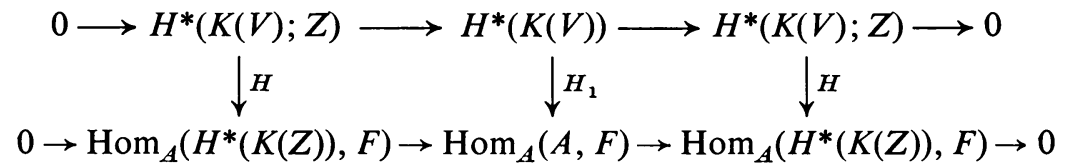

The top row is an exact triangle but the map $H^{*}(K(V) ; Z) \rightarrow H^{*}(K(V) ; Z)$ is multiplication by $p$ and $H^{*}(K(V) ; Z)$ is a $Z_{p}$-vector space i.e.

$$
H_{*}\left(K\left(Z_{p}\right) ; Z\right)=H_{*}\left(K(Z) ; Z_{p}\right)
$$

so applying the universal coefficient theorem we see that $H^{*}\left(K\left(Z_{p}\right) ; Z\right)$ is a $Z_{p}$-vector space, therefore so is $H^{*}(K(V) ; Z)=\Pi H^{*}\left(K\left(Z_{p}\right) ; Z\right)$. The bottom row is exact because $0 \rightarrow H^{*}(K(Z)) \rightarrow A \rightarrow H^{*}(K(Z)) \rightarrow 0$ is the cohomology exact sequence of the triangle $K(Z) \rightarrow K(Z) \rightarrow K\left(Z_{p}\right) \rightarrow$ $K(Z)$ and $F$ is injective. We have already noted that $H_{1}$ is an isomorphism, it follows that $H$ is an isomorphism.

Let $X$ be a locally finite spectrum such that $\Pi_{i}(X)=0$ for $i>I$. Then $\Pi_{*}(X)$ is finitely generated and we will let size $\Pi_{*}(X)$ equal the dimension of $\Pi_{*}(X) \otimes Q$ plus the cardinality of Tor $\Pi_{*}(X)$. Then there is an exact triangle $\Sigma^{k} K(G) \leftarrow X_{1} \leftarrow X \leftarrow \sum^{k} K(G)$ with $G$ either $Z_{q}$ for some prime $q$ or $Z$ and size $\Pi_{*}\left(X_{1}\right)<\operatorname{size} \Pi_{*}(X)$ (unless $X=K(G)$ already). Then we have the following diagram:

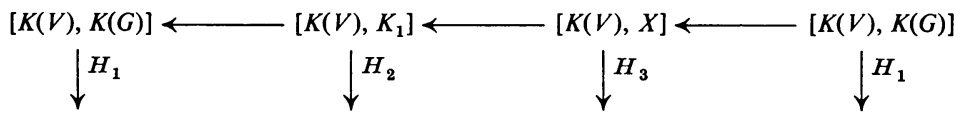

$\operatorname{Hom}_{A}\left(H^{*}(K(G)), F\right) \longleftarrow \operatorname{Hom}_{A}\left(H^{*}\left(X_{1}\right), F\right) \longleftarrow \operatorname{Hom}_{A}\left(H^{*}(X), F\right) \longleftarrow \operatorname{Hom}_{A}\left(H^{*}(K(G)), F\right)$ 
The top row is, in general, exact and, as above, $F$ injective implies that the bottom row is exact. Then by induction on size we conclude that $H_{3}$ is an isomorphism.

Let $X$ be a locally finite spectrum, then its Postnikov tower $X_{0} \leftarrow X_{1} \leftarrow \cdots X$ satisfies the condition that $\Pi_{*}\left(X_{r}\right)$ is finitely generated for each $r$. Therefore in the diagram

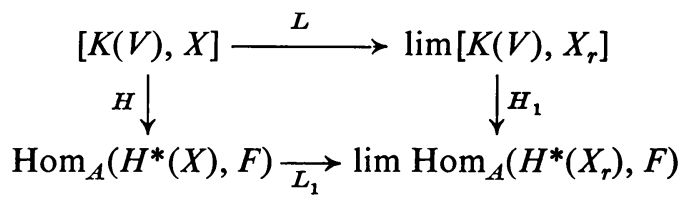

$H_{1}$ is an isomorphism. In general $L$ is an epimorphism and since $H^{*}(X)=$ proj $\lim H^{*}\left(X_{r}\right)$ the map $L_{1}$ is an isomorphism. Therefore $H$ is an epimorphism for any locally finite spectrum.

Proof of Theorem 2(a). Let $\alpha: H^{*}(X) \rightarrow N \oplus F$ be an isomorphism with $F$ free over $A$. Since $X$ is locally finite so is $F$ and therefore $F=H^{*}(K(V))$ where $V=Z_{p} \otimes_{A} F$ and further

$$
[X, K(V)] \rightarrow \operatorname{Hom}_{A}\left(H^{*}(K(V)), H^{*}(X)\right)
$$

is an isomorphism. Therefore there is an exact triangle $Y \rightarrow^{g} X \rightarrow^{f} K(V) \rightarrow Y$ with $f^{*}=\alpha^{-1} i_{2}$ and therefore $H^{*}(Y)=N$ and $g^{*}=\Pi_{1} \alpha$. We have proven that $[K(V), X] \rightarrow \operatorname{Hom}_{A}\left(H^{*}(X), H^{*}(K(V))\right)$ is epic and therefore there is a map $h: K(V) \rightarrow X$ such that $h^{*}=\Pi_{2} \alpha$. From this we get the commuting diagram:

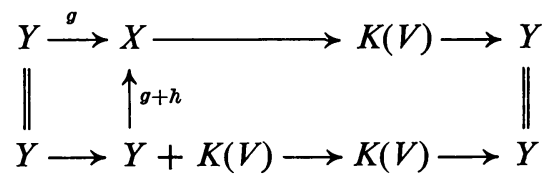

and there exists a map $k: K(V) \rightarrow K(V)$ filling in the diagram. But $(g+h)^{*}=$ $\alpha$ and therefore $k^{*}$ is an isomorphism. Since $\Pi_{*}(K(V))$ is $p$-primary, $k$ is an equivalence (if $K(V) \rightarrow{ }^{k} K(V) \rightarrow W \rightarrow K(V)$ is exact then $\Pi_{*}(W)$ is $p$-primary and $\left.H^{*}(W)=0\right)$, therefore $g+h$ is an equivalence.

Proof of THEOREM 2(b). By Theorem 1 there is an expression unique up to isomorphism $\alpha: H^{*}(X) \rightarrow N \oplus F$ with $N$ having no free summands. By Theorem 2(a) $X$ is equivalent to $Y \oplus K(V)$ with $H^{*}(Y)=N$ and $V=Z_{p} \otimes_{A} F$. Since $K(V)$ is determined up to equivalence by $V$ it remains to show that $Y$ is unique up to equivalence. So suppose we have equivalences $f: Y_{1} \oplus K(V) \rightarrow X$ and $g: Y_{2} \oplus K(V) \rightarrow X$ with $\alpha=f^{*}=g^{*}$. Then we get

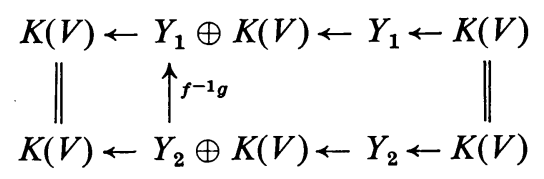


which commutes since $[W, K(V)] \rightarrow \operatorname{Hom}_{A}\left(H^{*}(K(V)), H^{*}(W)\right)$ is an isomorphism. Therefore a fill-in $h: Y_{2} \rightarrow Y_{1}$ exists and is an equivalence.

Proof of Theorem 3. It remains to show that

$$
[K(V), X] \rightarrow \operatorname{Hom}_{A}\left(H^{*}(X), F\right)
$$

is a monomorphism. Let us suppose that we are given $f: K(V) \rightarrow X$ with $f^{*}=0$ (in $Z_{p}$-cohomology). We have an exact triangle $K(V) \rightarrow^{f} X \rightarrow$ $Y \rightarrow K(V)$ which in $Z_{p}$-cohomology gives the short exact sequence $0 \rightarrow F \rightarrow H^{*}(Y) \rightarrow H^{*}(X) \rightarrow 0$. Therefore $H^{*}(Y)=H^{*}(X) \oplus F$ and by Theorem 2(a) there is an equivalence $g: Y \rightarrow X \oplus K(V)$. This gives commutative diagram

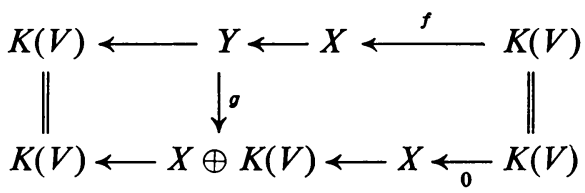

and so there is a fill-in $h: X \rightarrow X$. But $g$ an equivalence implies that $h$ is an equivalence and therefore $f=0$.

\section{BIBLIOGRAPHY}

1. J. F. Adams and H. R. Margolis, Modules over the Steenrod algebra, Topology 10 (1971), 271-282. MR 45 \#3520.

2. J. M. Boardman, Stable homotopy theory, Warwick lecture notes series (1965).

3. - Stable homotopy theory, Chap. II, preprint (1970).

4. H. R. Margolis, Modules over the Steenrod algebra and stable homotopy theory, (to appear).

5. J. C. Moore and F. P. Peterson, Nearly Frobenius algebras, Poincare algebras and their modules (preprint).

6. R. M. Vogt, Boardman's stable homotopy category, Lecture Note Series, no. 21, Aarhus Univ., Aarhus, 1970. MR 43 \#1187.

Department of Mathematics, Boston College, Chestnut Hill, Massachusetts 02167 